\title{
PERMUTATION LABELING OF JOINS OF KITE GRAPH
}

\author{
S. Sriram \\ Department of Mathematics, Patrician College of Arts and Science, \\ Adyar, Chennai-20, Tamil Nadu, India \\ Dr. R. Govindarajan \\ P. G and U. G, Department of Mathematics, D.G. Vaishnav College, \\ Arumbakkam, Chennai, Tamil Nadu, India
}

\begin{abstract}
Let $G=(V, E)$ be a graph with $p$ vertices and $q$ edges. A graph $G=\{V, E\}$ with $p$ vertices and $q$ edges is said to be a Permutation labelling graph if there exists a bijection function $f$ from set of all vertices $V(G)$ to $\{1,2,3 \ldots p\}$ such that the induced edge labelling function $h: E(G) \rightarrow N \quad$ is defined as $h\left(x_{1}, x_{2}\right)=f\left(x_{1}\right) P_{f\left(x_{2}\right)}$ or $f\left(x_{2}\right) P_{f\left(x_{1}\right)}$ according as $f\left(x_{1}\right) \prec f\left(x_{2}\right)$ or $f\left(x_{2}\right) \prec f\left(x_{1}\right)$ where $P$ is the permutation of objects(representing the labels assigned to vertices). We in this paper have identified $(m, n)$ Kite graph and attached an edge to form a join to the kite graph and proved that the joins of $(m, n)$ kite graphs is permutation labelling graph and also have obtained some important results connecting the joins of a $(m, n)$ kite graph.
\end{abstract}

Key words: (m, n) Kite Graph, Permutation Labelling of Graph, Joins of (m, n) Kite Graphs Mathematics Subject Classification: 05C78

Cite this Article: S. Sriram and Dr. R. Govindarajan, Permutation Labeling of Joins of Kite Graph. International Journal of Computer Engineering \& Technology, 10(3), 2019,pp.1-8. http://iaeme.com/Home/issue/IJCET?Volume $=10 \&$ Issue $=3$

\section{INTRODUCTION}

A graph $\mathrm{G}$ is a finite nonempty set of objects called vertices and edges. All graphs considered here are finite, simple and undirected. Gallian J. $\mathrm{A}^{[1]}$ has given a dynamic survey of graph labelling. The origin of graph labelings can be attributed to Rosa. The vertex set is denoted by $\mathrm{V}(\mathrm{G})$ and the edge set is denoted by $\mathrm{E}(\mathrm{G})$. Different labelling techniques have been a motivation and one such kind of labelling is the permutation labelling of graph discussed by Seoud M A, Salim M A ${ }^{[2]}$. Some more graphs have been labelled as permutation labelling ${ }^{[3]}$. In this paper we have identified the $(m, n)$ Kite graph and tried to attach edge to the $(m, n)$ Kite graphs to form a chain of $(m, n)$ Kite graphs which we call as Join of $(m, n)$ Kite Graph and we prove that the Join of Kite Graph is permutation labeling graph and we exhibit some important results 
connecting the joins of a $(m, n)$ kite graph. The discussion on the joins of $(m, n)$ Kite graph and the labelling of the vertices have been discussed while proving the graph as harmonic mean labelling graph ${ }^{[4]}$. The preliminary concepts of graphs we follow as in Handbook for graph ${ }^{[5]}$.

\section{PRELIMINARIES}

Definition. 2.1: A graph $G$ with $p$ vertices and $q$ edges is called a Permutation labelling if there exists a bijection function $\mathrm{f}$ from set of all vertices $V(G)$ to $\{1,2,3 \ldots p\}$ such that the induced edge labelling function $h: E(G) \rightarrow N$ is defined as $h\left(x_{1}, x_{2}\right)=f\left(x_{1}\right) P_{f\left(x_{2}\right)}$ or $f\left(x_{2}\right) P_{f\left(x_{1}\right)}$ according as $f\left(x_{1}\right) \prec f\left(x_{2}\right)$ or $f\left(x_{2}\right) \prec f\left(x_{1}\right)$

Definition.2.2: An $(m, n)$ Kite graph consists of cycle of length $m$ with $n$ edges path attached to one vertex of a cycle

We have worked on the following definition of attaching an edge from a $(m, n)$ kite graph to another $(m, n)$ kite graph and called it as 1-join of $(m, n)$ kite graph in ${ }^{[4]}$ which we have illustrated for the purpose of proving some results on permutation graph of $(m, n)$ Kite graph in this paper and also we have used the same labelling technique for assigning the 1-join of $(\mathrm{m}, \mathrm{n})$ kite graph as we have considered ${ }^{[4]}$ so as to prove that 1 -join of $(m, n)$ graph is permutation

Definition.2.3: For a $(m, n)$ Kite graph we attach an edge from the Kite graph to another $(m, n)$ Kite graph and we call the same as 1-Join of $(m, n)$ Kite graph.

The 1-Join of $(3,3)$ Kite graph is given as

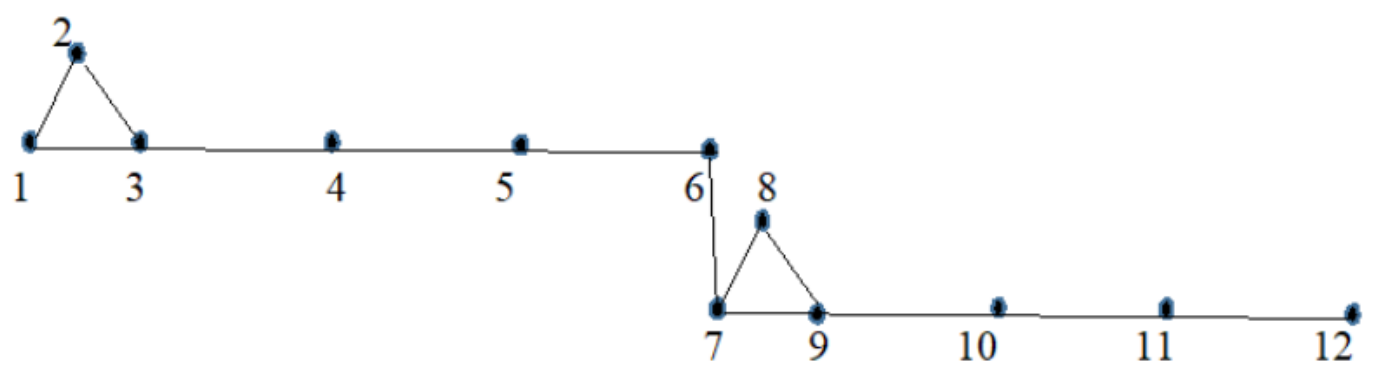

The above is a 1-join of $(3,3)$ Kite graph with a cycle of length 3 and with 3 edges path attached to one vertex of a cycle is joined by an edge with another $(3,3)$ Kite graph with a cycle of length 3 and with 3 edges path attached to one vertex of a cycle

Similarly we can construct 2-join $(m, n)$ Kite graph by attaching one more edge with another $(3,3)$ kite graph . Continuing this process we get a chain of Join of $(m, n)$ Kite graph.

Note: We here exclusively study the case of $\left(m_{1}, n_{1}\right)$ Kite graph attached by an edge $e_{1}$ to $\left(m_{2}, n_{2}\right)$ Kite graph,$\left(m_{2}, n_{2}\right)$ Kite graph attached by an edge $e_{2}$ to $\left(m_{3}, n_{3}\right)$ Kite graph and so on $\left(m_{n-1}, n_{n-1}\right)$ Kite graph attached by an edge $e_{n}$ to $\left(m_{n}, n_{n}\right)$ Kite graph respectively such that $m_{1}=m_{2}=m_{3}=\ldots=m_{n}$ and $n_{1}=n_{2}=n_{3}=\ldots=n_{n}$ where each $m_{i}$ is the cycle of same length and $n_{i}$ is the number of edges in the path of same length.

We discuss the varieties of Join of $(m, n)$ Kite graph as follows as we discussed in ${ }^{[4]}$ so as to preserve the case of discussion. 
Case.1 When $\mathrm{m}=\mathrm{n}$

Example: 1-Join $(3,3)$ Kite Graph with $(3,3)$ Kite graph

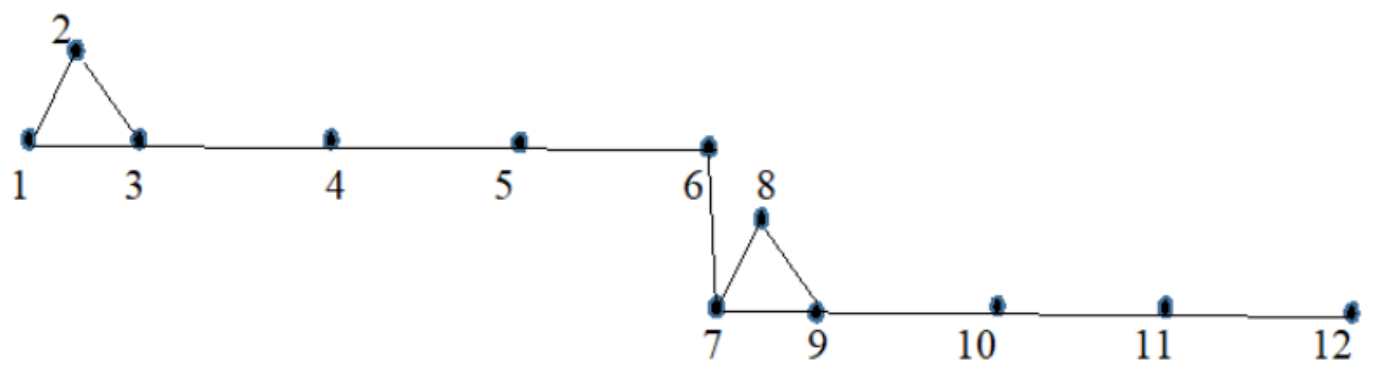

Case. 2 When $\mathrm{m}<\mathrm{n}$

Example: 1-Join $(3,4)$ Kite Graph with $(3,4)$ Kite graph

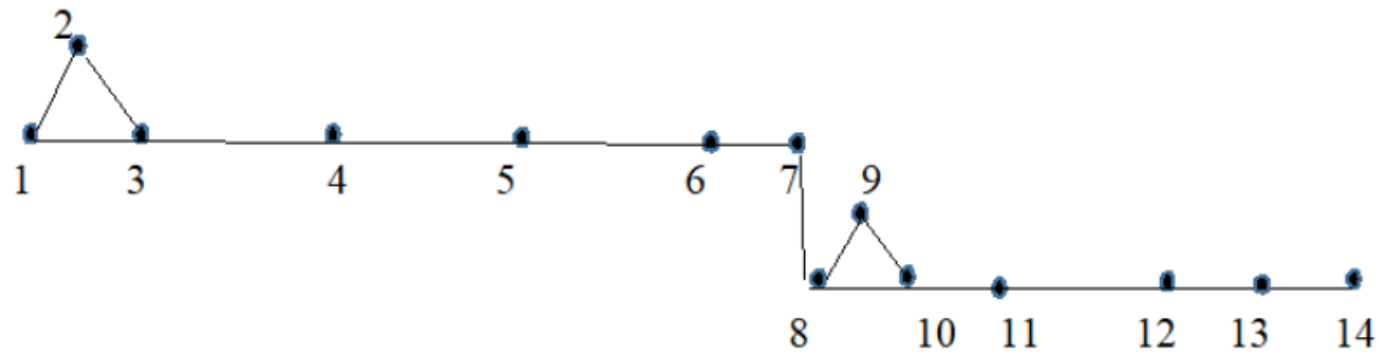

Case.3 When $\mathrm{m}>\mathrm{n}$

Example: 1-Join $(3,2)$ Kite Graph with $(3,2)$ Kite graph

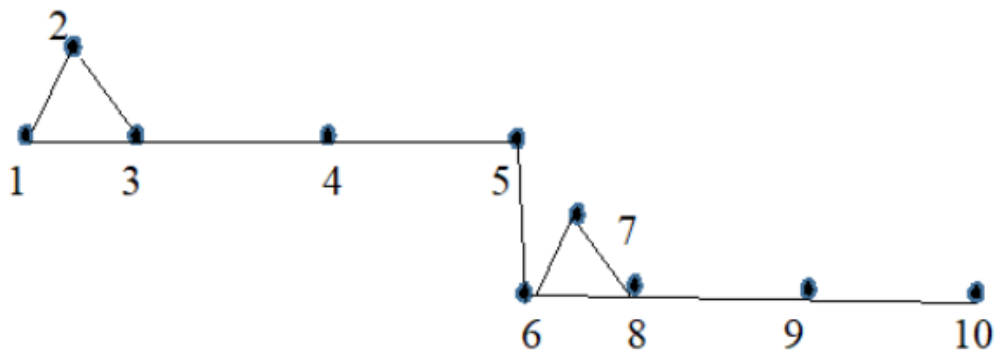

Note : In general we can consider any form of the above mentioned cases of $(m, n)$ Kite graph to join by an edge to form a chain of $(m, n)$ Kite graphs . Let us consider 1-Join of $(m, n)$ Kite graph as the basic graph and show that in the following theorem is a Permutation Labeling graph.

\section{MAIN RESULTS}

Theorem .3.1: The 1- Join of $(m, n)$ Kite graph $G$ is a Permutation Labeling graph.

Proof: Let $\mathrm{G}=1$-Join of $(\mathrm{m}, \mathrm{n})$ Kite graph

Let us prove that $\mathrm{G}$ is a Permutation labeling graph 
Let us prove the theorem by labelling the vertices of the graph for all the three cases as mentioned above we have the number of vertices in $(m, n)$ Kite graph as $m+n$ and the number of edges in $(m, n)$ Kite graph as $m+n$. Now adding one edge between the $(m, n)$ kite graph and another $(m, n)$ kite graph we have the number of vertices in 1-Join of $(m, n)$ Kite graph is $2(m+n)$ and the number of edges in 1-Join of $(m, n)$ Kite graph is $2(m+n)+1$.

Now we follow the following Scheme for labelling the vertices of $(m, n)$ Kite graph with another $(\mathrm{m}, \mathrm{n})$ kite graph

Total labels required to label the vertices of 1-Join $(m, n)$ Kite graph the is $2(m+n)$ and we label them as $1,2,3 \ldots 2(m+n)$.

Let us denote the Vertex Set of first (m,n) Kite graph as $V=\left\{u_{1}, u_{2}, u_{3}, u_{4}, \ldots u_{m+n}\right\}$ and the vertex set of second (m,n) Kite graph as $V^{1}=\left\{u_{1}^{1}, u_{2}^{1}, u_{3}^{1}, u_{4}^{1} \ldots u_{m+n}^{1}\right\}$. The edge set of first $(\mathrm{m}, \mathrm{n})$ Kite graph is $E=\left\{e_{1}, e_{2}, e_{3}, e_{4}, . . e_{m+n}\right\}$ and the edge of the second (m,n) Kite Graph is $E^{1}=\left\{e_{1}^{1}, e_{2}^{1}, e^{1}{ }_{3}, e^{1}{ }_{4} \ldots e^{1}{ }_{m+n}\right\}$. Now by adding one edge between the first and second (m,n) Kite graph we have the Vertex set of 1-Join $(m, n)$ Kite graph as

$V(G)=\left\{u_{1}, u_{2}, u_{3}, u_{4}, \ldots u_{m+n}\right\} \cup\left\{u_{1}^{1}, u_{2}^{1}, u_{3}^{1}, u_{4}^{1}{ }_{4} . u^{1}{ }_{m+n}\right\}$ and the edge set as

$E(G)=\left\{e_{1}, e_{2}, e_{3}, e_{4}, \ldots e_{m+n}\right\} \cup\left\{e^{1}{ }_{1}, e^{1}{ }_{2}, e^{1}{ }_{3}, e^{1}{ }_{4} . . e^{1}{ }_{m+n}\right\} \cup e^{1}$. The edges that are connecting

between the first and second $(\mathrm{m}, \mathrm{n})$ graph is known .

Now let us label the vertices of first $(m, n)$ kite graph in correspondence to the vertices of second $(\mathrm{m}, \mathrm{n})$ graph as follows

$$
\begin{aligned}
& f\left(u_{i}\right)=i \text { For } 1 \leq i \leq m+n \\
& f\left(u_{i}^{1}\right)=(m+n)+(i) \text { For } 1 \leq i \leq m+n
\end{aligned}
$$

Now we can compute the induced edge labelling as follows

$$
\begin{aligned}
& f^{*}\left(u_{i} u_{i+1}\right)=f\left(u_{i+1}\right) P_{f\left(u_{i}\right)} \\
& f^{*}\left(u_{i}^{1} u_{i+1}^{1}\right)=f\left(u_{i+1}^{1}\right) P_{f\left(u_{i}^{1}\right)} \\
& f^{*}\left(u_{m+n} u_{1}^{1}\right)=f\left(u_{1}^{1}\right) P_{f\left(u_{m+n}\right)}
\end{aligned}
$$

Hence the induced edge labelling can be found to be distinct and the labels that are assigned to edges of the graph $\mathrm{G}$ are permutation of the labels between the labels assigned for the vertices and result in natural numbers which are distinct and hence it can be claimed that the given graph $\mathrm{G}$ is Permutation labelling graph.

Hence the proof.

Theorem.3.2: M-Join of $(m, n)$ Kite graph is Permutation labelling graph.

Proof: Consider a M-Join of $(m, n)$ kite graph. To prove the graph is Permutation labelling graph. We know from theorem.3.1 that 1-join of $(m, n)$ kite graph is Permutation labeling graph. Now let us construct by adding 1 edge between the 1 -join of $(m, n)$ kite graph with a $(m, n)$ kite graph of the same order as of the kite graph in 1-join. The resulting graph is 2-join of $(m, n)$ kite graph and labeling as in theorem.3.1 we find that the induced edge labelling are permutation labeling and hence 2-join of $(m, n)$ kite graph is permutation labeling graph. Now continuing this procedure for any number of joins required say $\mathrm{M}$ we obtain $\mathrm{M}$-join of $(\mathrm{m}, \mathrm{n})$ kite graph and on labeling the vertices as in theorem.3.1 and as we know that the labels assigned for the vertices is consecutive numbers. The resulting M-Join of $(m, n)$ kite graph is permutation labeling graph. Hence the theorem. 
While construction of M-join we have assigned the labels for the vertices of first $(m, n)$ Kite graph as $u_{1}, u_{2}, u_{3} \ldots u_{m+n}$ and have assigned labels for the vertices of second (m,n) Kite graph as $u_{1}{ }^{1}, u_{2}{ }^{1}, u_{3}{ }^{1} \ldots u_{m+n}{ }^{1}$ and so on to construct joins of $(\mathrm{m}, \mathrm{n})$ kite graph . Now for obtaining some results on the $\mathrm{M}$-joins of $(m, n)$ Kite graph let us consider the vertices in continuous order as $u_{1}, u_{2}, u_{3} \ldots u_{m+n}, u_{m+n+1} \ldots u_{2(m+n)}, u_{2(m+n)+1, \ldots} u_{3(m+n)+1, \ldots}$. This procedure helps us in labelling the vertices continuously, but the assignment of labels for the vertices are followed as in Theorem.3.1.

Theorem.3.3: For a M-Join of $(m, n)$ Kite graph which is Permutation labelling graph then the following results holds good for $m=3$ and $n=2,3$ or $n=4$

(i) $\quad f^{*}\left(u_{i} u_{i+1}\right)=u_{i+1}$ for $1 \leq i \leq(m+n)-1$

(ii) If $m=n$ then

$f^{*}\left(u_{(M+1)(2 m)-(m+1)}, u_{(M+1)(2 m)-(m)}\right)=\mid(m-1)\left[f\left(u_{(M+1)(2 m)-(m+2)}\right) P_{f\left(u_{(M+1)(2 m)-(m)}\right)}\right]$

If $m \succ n$ that is for $n=m-1$

$f^{*}\left(u_{(M+1)(2 m-1)-(m-1)}, u_{(M+1)(2 m-1)-(m)}\right)=(m-1)\left[f\left(u_{(M+1)(2 m-1)-(m+1)}\right) P_{f\left(u_{(M+1)(2 m-1)-(m-1)}\right)}\right]$

If $m \prec n$ that is for $n=m+1$

$f^{*}\left(u_{(M+1)(2 m+1)-(m+1)}, u_{(M+1)(2 m+1)-(m)}\right)=(m-1)\left[f\left(u_{(M+1)(2 m+1)-(m+2)}\right) P_{f\left(u_{(M+1)(2 m+1)(m)}\right)}\right]$

Proof: Consider a M-Join of $(m, n)$ Kite graph which is Permutation labelling graph then to prove

$$
f^{*}\left(u_{i} u_{i+1}\right)=u_{i+1} \text { for } 1 \leq i \leq(m+n)-1
$$

The result is obvious from the definition of Permutation labelling between the vertices Consider $f^{*}\left(u_{i} u_{i+1}\right)=f\left(u_{i+1}\right) P_{f\left(u_{i}\right)}$, as we know the vertices are labelled with consecutive numbers we have by the well know formula of $n \mathrm{P}_{\mathrm{r}}=\frac{\underline{n}}{\underline{\underline{n}-r}}$ and here the labels of the vertices are $f\left(u_{i+1}\right)$ and $f\left(u_{i}\right)$. Hence on substituting we have $f\left(u_{i+1}\right) P_{f\left(u_{i}\right)}=\frac{\mid f\left(u_{i+1}\right)}{f\left(u_{i+1}\right)-f\left(u_{i}\right)}=f\left(u_{i+1}\right)$. Hence the proof for (i)

Now to claim the result (ii) for $\mathrm{m}=\mathrm{n}$ as

$$
f^{*}\left(u_{(M+1)(2 m)-(m+1)}, u_{(M+1)(2 m)-(m)}\right)=\underline{(m-1)}\left[f\left(u_{(M+1)(2 m)-(m+2)}\right) P_{f\left(u_{(M+1)(2 m)-(m)}\right)}\right]
$$

Let us consider L.H.S

$$
f^{*}\left(u_{(M+1)(2 m)-(m+1)}, u_{(M+1)(2 m)-(m)}\right)=\frac{\mid f\left(u_{(M+1)(2 m)-(m)}\right)}{f\left(u_{(M+1)(2 m)-(m)}\right)-f\left(u_{(M+1)(2 m)-(m+1)}\right)}
$$

Consider the R.H.S of the result 
$\underline{(m-1)}\left[f\left(u_{(M+1)(2 m)-(m+2)}\right) P_{f\left(u_{(M+1)(2 m)-(m)}\right)}\right]=\mid(m-1)\left[\frac{\mid f\left(u_{(M+1)(2 m)-(m+2)}\right)}{\mid f\left(u_{(M+1)(2 m)-(m+2)}\right)-f\left(u_{(M+1)(2 m)-(m)}\right)}\right]$ By choosing the number of Joins in the Kite graph that is $\mathrm{M}$ and value of $\mathrm{m}$ we can find that L.H.S=R.H.S

Hence the proof.

In a similar way for the result (ii) for $m \succ n$ by replacing $n=m-1$ and applying the permutation formula as we proved for $m=n$

We have the required result

$$
f^{*}\left(u_{(M+1)(2 m-1)-(m-1)}, u_{(M+1)(2 m-1)-(m)}\right)=(m-1)\left[f\left(u_{(M+1)(2 m-1)-(m+1)}\right) P_{f\left(u_{(M+1)(2 m-1)-(m-1)}\right)}\right]
$$

Hence the proof for result (ii) $m \succ n$

Similarly for the result (ii) for $m \prec n$ by replacing $n=m+1$ and applying the permutation formula as we proved for $m=n$

We have the required result

$$
f^{*}\left(u_{(M+1)(2 m+1)-(m+1)}, u_{(M+1)(2 m+1)-(m)}\right)=(m-1)\left[f\left(u_{(M+1)(2 m+1)-(m+2)}\right) P_{f\left(u_{(M+1)(2 n+1)(m)}\right)}\right]
$$

Hence the proof of the result (ii) for $m \prec n$

Result 1: Consider Example.1 given above for three different cases namely $\mathrm{m}=\mathrm{n}, m \succ n$ and $m \prec n$.

We can obtain the above proved result in theorem.3.3 as follows

For $\mathrm{m}=\mathrm{n}$ the result is

$$
f^{*}\left(u_{(M+1)(2 m)-(m+1)}, u_{(M+1)(2 m)-(m)}\right)=\underline{(m-1)}\left[f\left(u_{(M+1)(2 m)-(m+2)}\right) P_{f\left(u_{(M+1)(2 m)-(m)}\right)}\right]
$$

Now consider the case $1 \mathrm{~m}=3, \mathrm{n}=3$ and hence $\mathrm{m}=\mathrm{n}=3$, Choose $M=1$

$$
\begin{aligned}
& f^{*}\left(u_{(M+1)(2 m)-(m+1)}, u_{(M+1)(2 m)-(m)}\right)=f^{*}\left(u_{8}, u_{9}\right)=\lfloor 9=362880 \\
& \mid(m-1)\left[f\left(u_{(M+1)(m+n)-(m+2)}\right) P_{f\left(u_{(M+1)(m+n)-(m)}\right)}\right]=\left\lfloor 2\left[9 P_{7}\right]=2(181440)=362880\right.
\end{aligned}
$$

Similarly for $m \succ n$ and choosing $n=m-1$ and as in Case. $3 \mathrm{~m}=3, \mathrm{n}=2$ and let $\mathrm{M}=1$

We have $f^{*}\left(u_{(M+1)(2 m-1)-(m-1)}, u_{(M+1)(2 m-1)-(m)}\right)=f^{*}\left(u_{8}, u_{7}\right)=\underline{8}=40320$

$$
\mid(m-1)\left[f\left(u_{(M+1)(2 m-1)-(m+1)}\right) P_{f\left(u_{(M+1)(2 m-1)(m-1)}\right)}\right]=\left\lfloor 2\left[8 P_{6}\right]=2(20160)=40320\right.
$$

Similarly for $m \prec n$ and for $n=m+1$ we have from Case. $2 \mathrm{~m}=3, \mathrm{n}=4$ and let $\mathrm{M}=1$

$$
\begin{aligned}
& f^{*}\left(u_{(M+1)(2 m+1)-(m+1)}, u_{(M+1)(2 m+1)-(m)}\right)=f^{*}\left(u_{10}, u_{11}\right)=\underline{11}=39916880 \\
& (m-1)\left[f\left(u_{(M+1)(2 m+1)-(m+2)}\right) P_{f\left(u_{(M+1)(2 m+1)(m)}\right)}\right]=2\left[11 P_{9}\right]=2(2520)=2(19958440)=39916880
\end{aligned}
$$

Hence it can be claimed for any M-Joins of $(m, n)$ Kite graph. When $m=3, n=2,3,4$

Observation 1 In general for $m \prec n$, when $\mathrm{m}=3, \mathrm{n}=5,6 \ldots$ 


\begin{tabular}{|c|c|}
\hline For $n=m+2$ & $\begin{array}{c}f^{*}\left(u_{(M+1)(2 m+2)-(m+2)}, u_{(M+1)(2 m+2)-(m+3)}\right) \\
=(m-1)\left[f\left(u_{(M+1)(2 m+2)-(m+2)}\right) P_{f\left(u_{(M+1)(2 m+2)-(m+4)}\right)}\right]\end{array}$ \\
\hline For $n=m+3$ & $\begin{array}{c}f^{*}\left(u_{(M+1)(2 m+3)-(m+3)}, u_{(M+1)(2 m+3)-(m+4)}\right) \\
=(m-1)\left[f\left(u_{(M+1)(2 m+3)-(m+5)}\right) P_{f\left(u_{(M+1)(2 m+3)-(m+3)}\right)}\right.\end{array}$ \\
\hline
\end{tabular}

In general, for $m=n$

\begin{tabular}{|c|c|}
\hline For $m=n=4$ & $\begin{array}{c}f^{*}\left(u_{(M+1)(2 m)-(m+1)}, u_{(M+1)(2 m)-(m)}\right) \\
=(m-1)\left[f\left(u_{(M+1)(2 m)-(m+3)}\right) P_{f\left(\psi_{(M+)(2 m)(m)}\right)}\right]\end{array}$ \\
\hline For $m=n=5$ & $\begin{array}{c}f^{*}\left(u_{(M+1)(2 m)-(m+1)}, u_{(M+1)(2 m)-(m)}\right) \\
=(m-1)\left[f\left(u_{(M+1)(2 m)-(m+4)}\right) P_{f\left(u_{(M+1)(2 m)-(m)}\right)}\right]\end{array}$ \\
\hline For $m=n=6$ & $\begin{array}{c}f^{*}\left(u_{(M+1)(2 m)-(m+1)}, u_{(M+1)(2 m)-(m)}\right) \\
=(m-1)\left[f\left(u_{(M+1)(2 m)-(m+5)}\right) P_{f\left(u_{(M+1)(2 m)-(m)}\right)}\right]\end{array}$ \\
\hline & ontinue for all values of $m=n$. \\
\hline
\end{tabular}

In general for $m \prec n$

\begin{tabular}{|c|c|}
\hline For $m \prec n, \mathrm{~m}=4$, & $f^{*}\left(u_{(M+1)(2 m+1)-(m+1)}, u_{(M+1)(2 m+1)-(m+2)}\right)$ \\
$\mathrm{n}=5, \mathrm{n}=\mathrm{m}+1$ & $=\mid(m-1)\left[f\left(u_{(M+1)(2 m+1)-(m+4)}\right) P_{f\left(u_{(M+1)(2 m+1)(m+1)}\right)}\right]$ \\
\hline For $m \prec n, \mathrm{~m}=4$, & $f^{*}\left(u_{(M+1)(2 m+2)-(m+2)}, u_{(M+1)(2 m+2)-(m+3)}\right)$ \\
$\mathrm{n}=6, \mathrm{n}=\mathrm{m}+2$ & $=(m-1)\left[f\left(u_{(M+1)(2 m+2)-(m+5)}\right) P_{f\left(u_{(M+1)(2 m+2)(m+2)}\right)}\left[\begin{array}{l} \\
\text { For } m \prec n, \mathrm{~m}=4,\end{array}\right.\right.$ \\
\hline
\end{tabular}




\begin{tabular}{|c|c|}
\hline $\mathrm{n}=7, \mathrm{n}=\mathrm{m}+3$ & $=\underline{(m-1)}\left[f\left(u_{(M+1)(2 m+3)-(m+6)}\right) P_{\left.f\left(u_{(M+1)(2 m+3)(m+3)}\right)\right]}\right]$ \\
\hline & We can continue for all values of $m \prec n$ in a similar fashion \\
\hline
\end{tabular}

Also it can be noted that for all values of $m \succ n$ we can obtain the general result.

Theorem.3.4: For a M-Join of $(m, n)$ Kite graph the above result given in Theorem.3.3 (ii) the R.H.S grows as $2,3,4 \ldots$ when we find the induced edge labelling on the L.H.S for $m=3,4,5, \ldots$ Proof: We observe from the above theorem.3.3 result that the M-Join of $(m, n)$ kite graph is a permutation labelling graph with a significant property that according to the nature of size of the cycle denoted by $m$ the induced edge labelling on the R.H.S grows as $2,3,4 \ldots$. That is for $m=3$ the R.H.S of the result grows as $\underline{2}$ and for $m=4$ the R.H.S of the result grows as $\underline{3}$ and in general for $\mathrm{m}$ the cycle of the kite graph the R.H.S of the result grows as $\mid m-1$. Hence the proof.

\section{RESULTS}

In this paper we have considered M-Join of $(m, n)$ Kite graph and proved to be Permutation labelling graph and have established some results on the nature of induced edge that it generates by labelling the vertices. We also have identified that the same labelling technique which we have used to label the vertices to prove it Harmonic mean labelling [4] can be utilised for labelling the vertices to prove that it is permutation labelling graph.

\section{CONCLUDING REMARKS}

We are investigating on the some more graphs for which the labelling techniques can be applied to prove it is permutation labelling graph and identify some properties corresponding to the induced edge labelling of graphs.

\section{REFERENCES}

[1] Gallian. J.A, A Dynamic Survey of Graph Labeling, 2018; Twenty first edition

[2] Seoud M A, Salim M A, On permutation labelling, Journal of the Egyptian Mathematical Society, Volume 19, Issue 3, pp 134-136, October 2011

[3] Baig A Q, Imran M, Permutation labelling of graphs, Journal of Combinatorial Mathematics and Computing, May 2012

[4] Sriram S, Govindarajan R, A Study on Harmonic Mean labelling of Joins of Kite graph, International Journal of Scientific Research and Reviews, 8(1), pp 2879-2885, 2019

[5] Gross.J and. Yellen.J, Handbook of graph theory, CRC, Press: 2004 